\title{
QUASICONFORMAL AND HQC MAPPINGS BETWEEN LYAPUNOV JORDAN DOMAINS
}

\author{
VLADIMIR BOZIN AND MIODRAG MATELJEVIĆ
}

\begin{abstract}
Let $h$ be a quasiconformal (qc) mapping of the unit disk $\mathbb{U}$ onto a Lyapunov domain. We show that $h$ maps subdomains of Lyapunov type of $\mathbb{U}$, which touch the boundary of $\mathbb{U}$, onto domains of similar type. In particular if $h$ is a harmonic qc (hqc) mapping of $\mathbb{U}$ onto a Lyapunov domain, using it, we prove that $h$ is co-Lipschitz (co-Lip) on $\mathbb{U}$. This settles an open intriguing problem.
\end{abstract}

\section{INTRODUCTION}

Throughout the paper we consider the following setting $\left(\mathbb{U}_{q c}\right)$ : Let $h: \mathbb{U} \rightarrow D$ be a $K$-qc map, where $\mathbb{U}$ is the unit disk and suppose that $D$ is a Lyapunov domain(see Definition 1.1 below). If in addition $h$ is harmonic we say that $h$ satisfies the hypothesis $\left(\mathbb{U}_{h q c}\right)$. Under the hypothesis $\left(\mathbb{U}_{q c}\right)$ we prove that for every $a \in \mathbb{T}=$ $\{|z|=1\}$, there is a special Lyapunov domain $U_{a}$, of a fixed shape, in the unit disk $\mathbb{U}$ which touches $a$ and a special, convex Lyapunov domain $\operatorname{lyp}(D)_{b}^{-}$(see the subsection 3.2 the definition (v) before the the proof of Theorem 3.9 1 , of a fixed shape, in $D$, which touches $b=h(a)$, such that $\operatorname{lyp}(D)_{b}^{-} \subset h\left(U_{a}\right) \subset H_{b}$, where $H_{b}$ is a half-plane whose the boundary line contains $b$. We can regard this result as "a good local approximation of a qc mapping $h$ by its restriction to a special Lyapunov domain so that its codomain is locally convex". In addition, if $h$ is harmonic, using this result, we prove that $h$ is co-Lip on $\mathbb{U}$. This settles an open intriguing problem in the subject and can be regarded as a version of the Kellogg- Warschawski theorem for hqc. In order to discuss the subject we first need a few basic definitions (see Section 2 for more details).

Definition 1.1 (Lyapunov curves). (i) Throughout the paper by $\varepsilon, \epsilon, c, c_{1}, \varepsilon_{1}, \epsilon_{1}, \kappa, \kappa_{1}$ etc. we denote positive constants and by $\mu, \mu_{1}$ etc. constants in the interval $(0,1)$.

(ii) Suppose that $\gamma$ is a rectifiable, oriented, differentiable planar curve given by its arc-length parameterization $g$. If

$$
l_{1}=\operatorname{lyp}(\gamma)=\operatorname{lyp}(\gamma, \mu):=\sup _{t, s \in[0, l]} \frac{\left|g^{\prime}(t)-g^{\prime}(s)\right|}{|t-s|^{\mu}}<\infty
$$

2010 Mathematics Subject Classification. Primary 30C62, 31C05 .

Key words and phrases. Harmonic maps, quasi-conformal maps, quasihyperbolic-metric, biLipschitz maps,Lyapunov domains.

Research partially supported by MNTRS, Serbia, Grant No. 174032.

${ }^{1} \operatorname{lyp}(D)_{b}^{-}=T_{b}\left(D_{0}^{-}\right)$, and $D_{0}^{-}$is defined in the part (iii) of Proposition 3.5. 
we say that $\gamma$ is a $C^{1, \mu}$ curve. $C^{1, \mu}$ curves are also known as Lyapunov (we say also more precisely $\mu$-Lyapunov) curves. We call $\operatorname{lyp}(\gamma)$ the Lyapunov multiplicative constant. In this setting we say that $\gamma$ is $\left(\mu, l_{1}\right)$-Lyap (of order $\mu$ with multiplicative constant $l_{1}$ ). We say that a bounded planar domain $D$ is $\mu$-Lyapunov (respectively ( $\left.\mu, l_{1}\right)$-Lyap), $0<\mu<1$, if it is bounded by $\mu$-Lyapunov(( $\left.\mu, l_{1}\right)$-Lyap) curve $\gamma$. In this setting it is convenient occasionally to use $l_{1}=l_{1}(D)$ instead of lyp $(\gamma)$.

For a complex valued function defined on a domain in the complex plane $\mathbb{C}$, we use the notation $\lambda_{f}=l_{f}(z)=|\partial f(z)|-|\bar{\partial} f(z)|$ and $\Lambda_{f}(z)=|\partial f(z)|+|\bar{\partial} f(z)|$, if $\partial f(z)$ and $\bar{\partial} f(z)$ exist.

Note that $\operatorname{Lyp}(\varepsilon, c)$ is a special domain of Lyapunov type with two cusps and vertex at 0 .

Definition 1.2 (Elementary Lyapunov curves and Special Lyapunov domains). The curve $\gamma(c, \mu)=\gamma\left(c, \mu, r_{0}\right)$ is defined, in polar coordinates $(r, \varphi)$, by joining the curves $\varphi=c r^{\mu}$ and $\pi-\varphi=c r^{\mu}, 0 \leq r<r_{0}$, which share the origin(see Example 2.7 for more details). An arc $L$, which is isometric to the curve $\gamma(c, \mu)$ we call an elementary Lyapunov (more precisely $\mu$-Lyapunov) curve. If $A$ is the isometry we call $b=A(0)$ the vertex of $L$. If an $\operatorname{arc} C$ is a circle arc or elementary $\mu$-Lyapunov for some $0<\mu<1$ we call it an elementary Lyapunov arc.

For $\varepsilon, c>0$ and $c|\varepsilon|^{\mu}<\pi / 2$, we use the notation

(i) $L_{0}=L(\varepsilon)=\operatorname{Lyp}(\varepsilon, c, \mu)=\left\{w: c|w|^{\mu}<\arg (w)<\pi-c|w|^{\mu},|w|<\varepsilon\right\}$.

If this set is subset of $H, D$ it seems convenient to denote it shortly by $H_{0}, D_{0}$ respectively.

A special domain of Lyapunov type (with possible two cusps) is a convex domain whose the boundary consists of two elementary Lyapunov curves. If the part of boundary of a Lyapunov ( $\mu$-Lyapunov) domain is an elementary Lyapunov curve with vertex at $b$, we call it special Lyapunov( $\mu$-Lyapunov with elementary arc) domain with vertex at $b$.

Note that the curve $\gamma(c, \mu)$ is $C^{1, \mu}$ but it is not $C^{1, \mu_{1}}$ for $\mu_{1}>\mu$ (at the origin), and $\operatorname{Lyp}(\varepsilon, c, \mu)$ is a special domain of Lyapunov type with two cusps and vertex at 0 .

As an application of the Gehring-Osgood inequality [6, 30] concerning qc mappings and quasi-hyperbolic distances, in the particular case of punctured planes, we prove Proposition 2.8 (we refer to this result as $(\mathrm{GeOs})$ ), which roughly stated says that:

if $f$ is a $K$-qc mapping of the plane such that $f(0)=0, f(\infty)=\infty$ and $z_{1}, z_{2} \in \mathbb{C}^{*}$, then the measures of the convex angles between $f\left(z_{1}\right), f\left(z_{2}\right)$ and $z_{1}, z_{2}$ can be compared. Using this we prove the part (IV) of Theorem 3.5. we shortly refer this result as (S-0)), which can be considered as our main result, and Theorem 3.9 which is a global version of $(\mathrm{S}-0)$.

Theorem 3.9 gives an approximation of Lyapunov domains by special Lyapunov domains and it is a crucial result for the application to hqc mappings, stated here as: 
(S-1) Suppose that $D$ is a Lyapunov domain and $h: \mathbb{U} \rightarrow D$ is a qc homeomorphism. Then for every $a \in \mathbb{T}=\{|z|=1\}$, there is a special Lyapunov domain $U_{a}$, of a fixed shape, in the unit disk $\mathbb{U}$ which touches $a$ and a special, convex Lyapunov domain $\operatorname{lyp}(D)_{b}^{-}$, of a fixed shape, in $D$, which touches $b=h(a)$, such that $\operatorname{lyp}(D)_{b}^{-} \subset h\left(U_{a}\right) \subset H_{b}$, where $H_{b}$ is a halfplane whose the boundary line contains $b$. Using this we reduce the proof of co-Lip property

(L0) : if $h$ satisfies the hypothesis $\left(\mathbb{U}_{h q c}\right)$ then it is co-Lip,

to what we call locally convex case. In order to avoid confusion, note that in addition Theorem 3.9 states that there is a special, convex Lyapunov domain $\operatorname{lyp}(D)_{b}(\operatorname{see}$ Definition 3.6), of a fixed shape, in $D$, which touches $b=h(a)$, such that $h\left(U_{a}\right) \subset$ $\operatorname{lyp}(D)_{b} \subset H_{b}$ (see figure 1). But we do not use this part in the proof of (L0).

Set $d_{b}(w)=\operatorname{dist}\left(w, \operatorname{lyp}(D)_{b}^{-}\right)$, and for $a=h^{-1}(b), d_{b}^{\prime}(w)=\operatorname{dist}\left(w, h\left(U_{a}\right)\right)$. By an elementary argument one can prove:

(L1) If $w-b$ is in the direction of the normal vector $n_{b}$ of $\partial D$ at $b$, then $d_{b}(w) \approx$ $|w-b|$ if $|w-b|$ is small enough.

Note that the subject of hqc mappings has been intensively studied by the participants of the Belgrade Analysis Seminar (see Section2 for more details), in particular by Kalaj, who proved that if $h$ is a hqc mapping of the unit disk onto a Lyapunov domain, then $h$ is Lipschitz 12 (Kalaj also probably first posed the problem of whether $h$ is, in fact, bi-Lipschitz). Since there is a conformal mapping of the unit disk $\mathbb{U}$ onto a $C^{1}$ domain which is not Lipschitz, Kalaj's result is nearly optimal. In [17, it is shown that a harmonic diffeomorphism $h$ between two $C^{2}$ Jordan domains is a $\left(K, K^{\prime}\right)$ quasiconformal mapping for some constants $K \geq 1$ and $K^{\prime} \geq 0$ if and only if $h$ is bi-Lipschitz continuous (note that $(K, 0)$ qc is $K$-qc). These results naturally lead to the following question (conjecture):

Question 1. If $h: \mathbb{U} \rightarrow D$ is a hqc homeomorphism, where $D$ is a Lyapunov domain, is $h$ co-Lipschitz (shortly co-Lip)?

In Theorem 4.1 we give an affirmative answer to Question 1.

The following simple statements play an important role in the proof of Theorem 4.1 (co-Lip).

Proposition 524 states that if $h$ is a harmonic univalent orientation preserving $K$-qc mapping of domain $D$ onto $D^{\prime}$, then $d(z) \Lambda_{h}(z) \approx d_{h}(z), z \in D$. We need only a corollary of this:

(S-2) $d(z) \Lambda_{h}(z) \succeq d_{h}(z), z \in D$.

Using a slightly modification of the proof of Theorem 1.1 [24] (planar case) and Kellogg's theorem we can derive

(S-3) Suppose that $h$ is a euclidean harmonic mapping from a Lyapunov domain $G$ into a domain $D$ and there is a half space $H_{b}$ which touches a point $b \in \partial D$ such that $D=h(G) \subset H_{b}$. Then $d(h(z), b) \succeq d_{G}(z), z \in G$.

We say that a domain $\mathrm{D}$ is locally convex at a point $b \in \partial D$ if there is a half space $H_{b}$ such that $D \subset H_{b}$.

For the convenience of the reader we summarize that $(\mathrm{S}-1),(\mathrm{S}-2)$ and $(\mathrm{S}-3)$, are the main ingredients in the proof of Theorem 4.1 stated here as 
Theorem 1.3. Suppose $h: \mathbb{U} \rightarrow D$ is a hqc homeomorphism, where $D$ is a Lyapunov domain with $C^{1, \mu}$ boundary. Then $h$ is co-Lipschitz.

Remark 1.4. Note that, in general, $h\left(U_{a}\right)$ is not convex and we can not apply our consideration [24] (see the proof of Theorem 1.2 there) directly; but $h\left(U_{a}\right) \subset H_{b}$ is locally convex at $b$ and we can apply (S3) (note that we do not use the fact that $\operatorname{lyp}(D)_{b}^{-}$is a convex Lyapunov domain).

Recall that a mapping $h$ which is $\left(\mathbb{U}_{q c}\right)$ satisfies $(\mathrm{S}-1)$. If $h$ is in addition harmonic then we can apply (S-3). This is crucial for the proof of theorem and it reduces the proof to the locally-convex case.

Definition 1.5 (Hypothesis $\left(\mathbb{H}_{q c}\right),\left(\mathbb{H}_{h q c}\right),\left(\mathbb{H}_{q c}^{0}\right),(\mathrm{H}-0),\left(\mathbb{U}_{q c}\right),\left(\mathbb{U}_{h q c}\right)$ and $\left.(\mathrm{U}-1)\right)$. It is convenient to consider the following hypothesis

(Sp0): $0 \in \partial D$ and $D$ has the real axis as a tangent at 0 , with inner normal pointing upwards.

$\left(\mathbb{H}_{q c}\right)$ : Let $h: \mathbb{H} \rightarrow D$ be $K$-qc map, where $\mathbb{H}$ is the upper -half plane and suppose $D$ is a Lyapunov domain with the boundary boundary $\partial D$ positively oriented.

Using rotation and translation, we can reduce the study of the behaviour of $h$ at a point $a \in \mathbb{R}$ to the following setting

(Lyp-0): $D$ is a Lyapunov domain and satisfies (Sp0).

If, in addition to $\left(\mathbb{H}_{q c}\right), h$ is harmonic, we say that $h$ satisfies the hypothesis $\left(\mathbb{H}_{h q c}\right)$. If $h$ satisfies the hypothesis $\left(\mathbb{H}_{q c}\right)$ (respectively $\left(\mathbb{H}_{h q c}\right)$ ), $D$ satisfies the hypothesis (Lyp-0) and $h(0)=0$, we say that $h$ satisfies the hypothesis $\left(\mathbb{H}_{q c}^{0}\right)$, (respectively $(\mathrm{H}-0)$ ).

If $\mathbb{H}$ is replaced by $\mathbb{U}$ we denote the corresponding hypotheses by $\left(\mathbb{U}_{q c}\right),\left(\mathbb{U}_{h q c}\right)$; and $\mathbb{U}_{q c}^{0}($ respectively $\mathrm{U}-1)$ if in addition $h(1)=0$.

Note that in this paper we consider only the planar case. The plan of the exposition is as follows: In Section 2, we consider the background, definitions and basic properties of Lyapunov domains and we prove Proposition 2.8, which may be considered to be a version of the Gehring-Osgood inequality related to the measures of the corresponding angles. In Section 3 , we prove Theorem 3.5 and Theorem 3.9. In Section 4 we give the proof of Theorem 4.1(co-Lip).

The second author communicated the main result of this paper at CMFT 2017. ${ }^{2}$

We also suggest to the interested reader to make rough picture and scheme with corresponding notations in order to follow the manuscript; and first to read Section 3 without proofs and and then Section 4 with all details and finally to consider complete proofs and technical details in Section 3.

\section{BACKGROUND}

The next example which is shortly discussed in [8, 25, shows that there is a conformal map of unit disk onto $C^{1}$ domain which is not bi-Lipschitz.

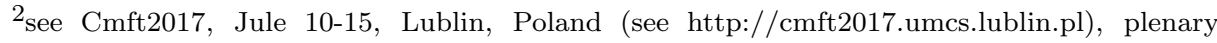
speakers.
} 
Example 2.1. Set

$$
w=A(z)=\frac{z}{\ln \frac{1}{z}}, \quad w(0)=0 .
$$

Note $\ln \frac{1}{z}=-\ln z, w^{\prime}(z)=-(\ln z)^{-1}+(\ln z)^{-2}$ and $w^{\prime}(z) \rightarrow 0$ if $z \rightarrow 0$ throughout $\mathbb{H}$. For $r$ small enough $A$ is univalent in $U_{r}^{+}$. We can check that there is a smooth domain $D \subset U_{r}^{+}$such that interval $\left(-r_{0}, r_{0}\right), r_{0}>0$, is a part of the boundary of $D, D^{*}=A(D)$ is $C^{1}$ domain and $A$ is not co-Lipschitz on $D$.

For basic properties of qc mappings the reader can consult Ahlfors's lovely book 4. Let $\gamma$ be a Jordan curve. By the Riemann mapping theorem there exists a Riemann conformal mapping of the unit disk onto the Jordan domain $G=\operatorname{int} \gamma$. By Caratheodory's theorem it has a continuous extension to the boundary. Moreover, if $\gamma \in C^{n, \alpha}, n \in \mathbb{N}, 0 \leq \alpha<1$, then the Riemann conformal mapping has a $C^{n, \alpha}$ extension to the boundary (this result is known as Kellogg's theorem), see 32. Conformal mappings are quasiconformal and harmonic. Hence quasiconformal harmonic (abbreviated by HQC) mappings are a natural generalization of conformal mappings.

Remark 2.2. Note that

a) The proof of Kellogg's theorem for conformal mapping is not elementary and it is based on some techniques which we can not adapt for hqc.

b)Since there is a conformal map of unit disk onto $C^{1}$ domain which is not biLipschitz (Example 2.1 above), it seems that the hypothesis that domains are Lyapunov is essential.

By a) and b) in mind, it seems that we need new approaches to study hqc mappings.

Recall that HQC mappings are now a very active area of investigation and some new methods have been developed for studying this subject (see for example 26] and literature cited there). Concerning the background we mention only a few results which are closely related to our results:

It seems that O. Martio 22] was the first one who considered HQC mapping of the unit disk and M. Pavlović proved in [29] that it is Lipschitz. An asymptotically sharp variant have been obtained by Partuka and Sakan [27. Among other things Knežević and the second author in [18] showed that a $K$-qc harmonic mapping of the unit disk onto itself is a $(1 / K, K)$ quasi-isometry with respect to the Poincaré and Euclidean metrics. For bi-lipschitz approximations of quasiconformal maps see Bishop [5]. M. Mateljević [24] and V. Manojlović [19] showed that hqc mappings are Bi-Lipschitz with respect to quasi hyperbolic metrics. Since the composition of a harmonic mapping and a conformal mapping is itself harmonic, using the case of the unit disk and Kellogg's theorem, these theorems can be generalized to the class of mappings from arbitrary Jordan domains with Lyapunov boundary onto the unit disk. However the composition of a conformal and a harmonic mapping is not, in general, a harmonic mapping. This means in particular, that results of this kind for arbitrary image domains do not follow directly from the case in which the codomain is the unit disk or the upper half-plane and Kelloggs theorem. In [16, Kalaj and the second author show how to combine Kellogg's theorem with the so called inner type estimate and that the simple proof in the case of the upper half-plane has an 
analogue for $C^{2}$ domains; namely, they proved a version of the "inner estimate" for quasi-conformal diffeomorphisms, which satisfies a certain estimate concerning their Laplacian. As an application of this estimate, it is shown that quasi-conformal harmonic mappings between smooth domains (with respect to the approximately analytic metric), have bounded partial derivatives; in particular, these mappings are Lipschitz. The discussion in 16 includes harmonic mappings with respect to (a) spherical and Euclidean metrics (which are approximately analytic) as well as (b) the metric induced by the holomorphic quadratic differential.

Although the following two statements did not get attention immediately after their publications, it turns out, surprisingly, that they play an important role in the proof of Theorem 4.1 (co-Lip).

Proposition 2.3 (Corollary 1, Proposition 5 [24]; see also [19]). Every e-harmonic quasi-conformal mapping of the unit disc (more generally of a strongly hyperbolic domain) is a quasi-isometry with respect to the hyperbolic distance.

Theorem 2.4 ([23]). Suppose that $h=f+\bar{g}$ is a Euclidean orientation preserving harmonic mapping from $\mathbb{U}$ onto the bounded convex domain $D=h(\mathbb{U})$, which contains a disc $B\left(h(0) ; R_{0}\right)$.

(I) Then $\left|f^{\prime}\right| \geq R_{0} / 4$ on $\mathbb{U}$.

(II) Suppose, in addition, that $h$ is qc. Then $l_{h} \geq(1-k)\left|f^{\prime}\right| \geq(1-k) R_{0} / 4$ on $\mathbb{U}$.

(III) In particular, $h^{-1}$ is Lipschitz.

See also Partyka and Sakan 28 .

Concerning the Lipschitz property of hqc, Kalaj [12] proved:

Theorem 2.5. Suppose $h: D_{1} \rightarrow D_{2}$ is a hqc homeomorphism, where $D_{1}$ and $D_{2}$ are domains with $C^{1, \mu}$ boundary.

(I) Then $h$ is Lipschitz.

(II) If, in addition, $D_{2}$ is convex, then $h$ is bi-Lipschitz.

With this theorem in mind Question 1 is natural. The proof of part (a) of Theorem 2.5 in 12 is based on an application of Mori's theorem on quasiconformal mappings, which has also been used in [29] in the case $D_{1}=D_{2}=\mathbb{U}$, and a geometric lemma related to Lyapunov domains.

2.1. Notation. Here we give a few basic definitions.

Definition 2.6 (qc). (i) By $\mathbb{C}$ we denote the the complex plane and by $\mathbb{T}$ the unit circle. For $r>0$ and $w \in \mathbb{C}$, we denote by $B(w, r)$ and the $C(w, r)$ the disk and circle of radius $r$ with center at $w$.

(ii) By $\mathbb{C}^{*}$ we denote the punctured complex plane $\mathbb{C} \backslash\{0\}$, by $\mathbb{H}^{*}$ the lower half plane $\{z: \operatorname{Im} z<0\}$ and by $\mathbb{U}^{+}$the upper half disk $\{z: \operatorname{Im} z>0,|z|<1\}$.

(iii) Recall that, for a complex valued function $h$ defined on a domain in the complex plane $\mathbb{C}$, we use the notation

$$
\lambda_{h}=l_{h}(z)=|\partial h(z)|-|\bar{\partial} h(z)| \quad \text { and } \quad \Lambda_{h}(z)=|\partial h(z)|+|\bar{\partial} h(z)|,
$$


if $\partial h(z)$ and $\bar{\partial} h(z)$ exist. A homeomorphism $h: D \rightarrow G$, where $D$ and $G$ are subdomains of the complex plane $\mathbb{C}$, is said to be $K$-quasiconformal ( $K$-qc or $k$-qc), $K \geq 1$, if $f$ is absolutely continuous on a.e. horizontal and a.e. vertical line in $D$ and there is $k \in[0,1)$ such that

$$
\left|h_{\bar{z}}\right| \leq k\left|h_{z}\right| \quad \text { a.e. on } D,
$$

where $K=\frac{1+k}{1-k}$, i.e. $k=\frac{K-1}{K+1}$.

Note that the condition (2.1) can be written as

$$
D_{h}:=\frac{\Lambda_{h}}{\lambda_{h}}=\frac{\left|h_{z}\right|+\left|h_{\bar{z}}\right|}{\left|h_{z}\right|-\left|h_{\bar{z}}\right|} \leq K
$$

where $K=\frac{1+k}{1-k}$, i.e. $k=\frac{K-1}{K+1}$.

(iv) Let $\Omega \subset \mathbb{R}^{n}$ and $\mathbb{R}^{+}=[0, \infty)$ and $f, g: \Omega \rightarrow \mathbb{R}^{+}$. If there is a positive constant $c$ such that $f(x) \leq c g(x), x \in \Omega$, we write $f \preceq g$ on $\Omega$. If there is a positive constant $c$ such that

we write $f \approx g$ (or $f \approx g$ ) on $\Omega$.

$$
\frac{1}{c} g(x) \leq f(x) \leq c g(x), \quad x \in \Omega
$$

To gain some intuition about Lyapunov curves we give a basic example:

Example 2.7. For $c>0,0<\mu<1$, and $x_{0}>0$, the curve $f(c, \mu)=f\left(c, \mu, x_{0}\right)$ in the $x y$-plane which is defined by

$$
\text { (1) } \quad y=c|x|^{1+\mu},|x|<x_{0} \text {, }
$$

is $C^{1, \mu}$ at the origin but is not $C^{1, \mu_{1}}$ for $\mu_{1}>\mu$. It is convenient to write this equation using polar coordinates $z=r e^{i \varphi}$ in the form: $r \sin \varphi=c r^{1+\mu}(\cos \varphi)^{1+\mu}$. Next, if $0 \leq \varphi \leq \pi / 2$, we have $\sin \varphi=\operatorname{cr}^{\mu}(\cos \varphi)^{1+\mu}, 0 \leq r<r_{0}$, where $r_{0}$ is a positive number. Since $\sin \varphi=\varphi+o(\varphi)$ and $\cos \varphi=1+o(1)$, we find $\varphi=c r^{\mu}+o(1)$ when $\varphi \rightarrow 0$. If $\pi / 2 \leq \varphi \leq \pi$, we have $\sin (\pi-\varphi)=\sin \varphi=c r^{\mu}(\cos \varphi)^{1+\mu}$, $0 \leq r<r_{0}$, where $r_{0}$ is a positive number. Since $\sin (\pi-\varphi)=\pi-\varphi+o(\pi-\varphi)$ and $\cos \varphi=-1+o(1)$, we find $\pi-\varphi=c r^{\mu}+o(1)$ when $\varphi \rightarrow \pi$. The curve $\gamma(c, \mu)=$ $\gamma\left(c, \mu, r_{0}\right)$ defined by joining the curves $\varphi=c r^{\mu}$ and $\pi-\varphi=c r^{\mu}, 0 \leq r<r_{0}$, which share the origin, has similar properties near the origin to the curve defined by (1). The reader can check that the curves $f(c, \mu)$ and $\gamma(c, \mu)$ are $C^{1, \mu}$ at the origin but are not $C^{1, \mu_{1}}$ for $\mu_{1}>\mu$.

Note that if a curve satisfies $\varphi \leq c r^{\mu}$, then it is is below the curve $\gamma(c, \mu)$.

2.2. Gehring-Osgood inequality. We can compute the quasihyperbolic metric $k$ on $\mathbb{C}^{*}$ by using the covering exp : $\mathbb{C} \rightarrow \mathbb{C}^{*}$, where exp is the exponential function. Let $z_{1}, z_{2} \in \mathbb{C}^{*}, z_{1}=r_{1} e^{i t_{1}}, z_{2}=r_{2} e^{i t_{2}}$ and $\theta=\theta\left(z_{1}, z_{2}\right) \in[0, \pi]$ the measure of the convex angle between $z_{1}, z_{2}$. We use

$$
k\left(z_{1}, z_{2}\right)=\sqrt{\left|\ln \frac{r_{2}}{r_{1}}\right|^{2}+\theta^{2}} .
$$

This well-known formula is due to Martin and Osgood. 
Let $\ell=\ell\left(z_{1}\right)$ be the line defined by 0 and $z_{1}$. Then $z_{2}$ belongs to one half-plane, say $M$, on which $\ell=\ell\left(z_{1}\right)$ divides $\mathbb{C}$.

Locally, denote by $\ln$ a branch of Log on $M$. Note that $\ln$ maps $M$ conformally onto a horizontal strip of width $\pi$. Since $w=\ln z$, we find that the quasi-hyperbolic metric

$$
|\mathrm{d} w|=\frac{|\mathrm{d} z|}{|z|} .
$$

Note that $\rho(z)=\frac{1}{|z|}$ is the quasi-hyperbolic density for $z \in \mathbb{C}^{*}$ and therefore

$$
k\left(z_{1}, z_{2}\right)=\left|w_{1}-w_{2}\right|=\left|\ln z_{1}-\ln z_{2}\right| .
$$

Let $z_{1}, z_{2} \in \mathbb{C}^{*}, w_{1}=\ln z_{1}=\ln r_{1}+i t_{1}$. Then $z_{1}=r_{1} e^{i t_{1}}$ and there is $t_{2} \in\left[t_{1}, t_{1}+\pi\right)$ or $t_{2} \in\left[t_{1}-\pi, t_{1}\right)$ such that $w_{2}=\ln z_{2}=\ln r_{2}+i t_{2}$. Hence

$$
k\left(z_{1}, z_{2}\right)=\sqrt{\left|\ln \frac{r_{2}}{r_{1}}\right|^{2}+\left(t_{2}-t_{1}\right)^{2}} .
$$

Now using the quasi-hyperbolic distance $k$ as a corollary of the Gehring-Osgood inequality, we can prove the following result which we will need.

Proposition 2.8. Let $f$ be a $K$-qc mapping of the plane such that $f(0)=0, f(\infty)=$ $\infty$ and $\alpha=K^{-1}$. If $z_{1}, z_{2} \in \mathbb{C}^{*},\left|z_{1}\right|=\left|z_{2}\right|$ and $\theta \in[0, \pi]$ (respectively $\theta^{*} \in[0, \pi]$ ) is the measure of the convex angle between $z_{1}, z_{2}$ (respectively $f\left(z_{1}\right), f\left(z_{2}\right)$ ), then

$$
\theta^{*} \leq c \max \left\{\theta^{\alpha}, \theta\right\}
$$

where $c=c(K)$. In particular, if $\theta \leq 1$, then $\theta^{*} \leq c \theta^{\alpha}$.

Proof. By the Gehring-Osgood inequality,

$$
k\left(f\left(z_{1}\right), f\left(z_{2}\right)\right) \leq c \max \left\{k\left(z_{1}, z_{2}\right)^{\alpha}, k\left(z_{1}, z_{2}\right)\right\},
$$

where $c=c(K)$. It is clear that $\theta^{*} \leq k\left(f\left(z_{1}\right), f\left(z_{2}\right)\right)$. Since $\left|z_{1}\right|=\left|z_{2}\right|$ and $k\left(z_{1}, z_{2}\right)=t_{2}-t_{1}=\theta$, we get the desired result.

\section{MAIN RESUlt}

We first need some definitions.

Elementary Lyapunov domains, Arc-chord constant $b_{\gamma}$ and the second Lyapunov constant $l_{D}^{2}=l_{\gamma}^{2}$.

Definition 3.1 (Elementary Lyapunov domains). (i) Recall for $r>0$ and $w \in \mathbb{C}$, we denote by $B(w, r)$ and the $C(w, r)$ the disk and circle of radius $r$ with center at $w$. In particular, we use notation $B(r)$ and the $C(r)$ for the disk and circle of radius $r$ with center at 0 and we denote by $C^{+}(r)$ the half circle in the upper half plane.

(ii)Definition of $L_{b}^{-}(\varepsilon)$. Further for $v>0$ let the circle $C(i v, r)$ touch the curve $\gamma=\gamma(\mu, c)$ at points $w_{1}$ and $w_{2}$ (say that $u_{1}<u_{2}$ ) and let $l^{+}$be the upper half arc of the circle $C(i v, r)$ joining $w_{1}$ and $w_{2}$ and $\gamma_{1}$ be the part of $\gamma$ over $\left[u_{1}, u_{2}\right]$, where $u_{k}=\operatorname{Re} w_{k}, k=1,2$. Then the domain enclosed by $l^{+}$and $\gamma_{1}$ we denote by 
$\operatorname{Lyp}(r, c)$. If $\epsilon^{0}$ is maximum of $r>0$ for which $\operatorname{Lyp}(r, c)$ belongs to $L(\varepsilon)$, we denote

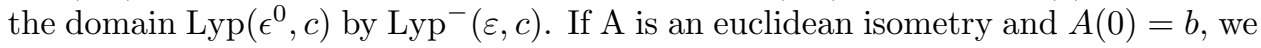
denote the domain $A\left(\operatorname{Lyp}^{-}(\varepsilon, c)\right)$ by $L_{b}^{-}(\varepsilon)=\operatorname{Lyp}_{b}^{-}(\varepsilon, c)$ and call it an elementary $\mu$ - Lyapunov domain.

Although the boundary of an elementary Lyapunov domain consists of an elementary $\mu$ - Lyapunov arc $\gamma_{0}$ and a circle arc $C_{0}$ with common end points, say $a_{0}$ and $b_{0}$ note that it has no cusps because $\gamma_{0}$ and $C_{0}$ have common tangents at points $a_{0}$ and $b_{0}$.

Definition 3.2 (arc-chord condition). More generally, if we suppose only that the curve is rectifiable we can define the distance along it. Let $C$ be a rectifiable Jordan closed curve and $z_{1}, z_{2}$ finite points of $C$. They divide $C$ into two arc, and we consider one with smaller Euclidean length and denote its length with $d_{C}\left(z_{1}, z_{2}\right)$.

(a) The curve $C$ is said to satisfy the arc-chord condition if the ratio of this length to the distance $\left|z_{1}-z_{2}\right|$ is bounded by a fixed number $b_{C}=b_{C}^{a r c}$ (which we call arc-chord constant of $C$ ) for all finite $z_{1}, z_{2} \in C$.

(b) The curve $C$ is said to satisfy the arc-chord condition at a fixed point $z_{1} \in C$ if the ratio of the length $d_{C}\left(z_{1}, z\right)$ to the distance $\left|z_{1}-z\right|$ is bounded by a fixed number $b_{C}\left(z_{1}\right)=b_{C}^{\text {arc }}\left(z_{1}\right)$ for all finite $z \in C$.

(c) If $D$ is a $\mu$-Lyapunov domain bounded by a curve $\gamma$, we define $l_{2}=l_{2}(D)=$ $l_{D}^{2}=l_{\gamma}^{2}=\frac{\pi}{2} l_{1} b_{\gamma}^{1+\mu}$, and we call it the second Lyp-constant, where $l_{1}=$ $\operatorname{lyp}(\gamma, \mu)$.

3.1. Auxiliary results. Suppose that $D$ satisfies the hypothesis (Lyp-0). Further, one can prove

(c1) It is known that that a $C^{1}$ curve satisfies the arc-chord condition.

(d) there is $r_{1}>0$ such $\partial D \cap B\left(r_{1}\right)$ is graph of a function $F, v=F(u)$, $-u_{1}<u<u_{2}$, where $u_{1}, u_{2}>0$, and that the set $V=\left\{(u, v):-u_{1}<u<\right.$ $\left.u_{2}, F(u)<v\right\} \cap B\left(r_{1}\right)$ belongs $D$, where $u, v$ are the cartesian coordinates in w-plane and $w=u+i v$.

(e) Let $D$ be a bounded Lyapunov domain, $a_{0} \in D$ and let $\psi$ be a conformal mapping of $D$ onto $\mathbb{U}$ with $\psi\left(a_{0}\right)=0$. Then there are constants $k_{1}=$ $\underline{k}_{1}\left(D, a_{0}\right)$ and $k_{2}=\underline{k}_{2}\left(D, a_{0}\right)$ (which we call the lower and upper Kellogg multiplicative constants of $D$ with respect to $a_{0}$ respectively) such that $k_{1}\left|z_{1}-z_{2}\right| \leq\left|\psi\left(z_{1}\right)-\psi\left(z_{2}\right)\right| \leq k_{2}\left|z_{1}-z_{2}\right|, z_{1}, z_{2} \in D$.

(f) Mori's theorem. Let $f: \mathbb{U} \rightarrow \mathbb{U}$ be a surjective K-qc mapping with $f(0)=0$ and $\alpha=1 / K$. Then $\left|f\left(z_{1}\right)-f\left(z_{2}\right)\right| \leq 16\left|z_{1}-z_{2}\right|^{\alpha}, z_{1}, z_{2} \in \mathbb{U}$, i.e. $f$ is $\alpha$-Holder continuous.

(g) Let the mapping $A$ is given by $A(z)=i \frac{z-i}{z+i}+i$. Then $A(i)=i$ and $A$ maps $\mathbb{H}$ onto $B_{1}=B(i, 1)$. Since $A^{\prime}(z)=\frac{2}{|z+i|^{2}}$, we first find $\left|A^{\prime}(z)\right| \leq 2$ and therefore $|A(z)| \leq 2|z|, z \in \mathbb{H}$.

(h) Suppose that $D$ is a bounded convex planar domain, $f: \bar{D} \rightarrow \mathbb{C}$ is holomorphic mapping and $z_{0} \in D$. Then there is a constant $c>0$ such that $\left|f z-f z_{0}\right| \leq c\left|z-z_{0}\right|, z \in D$. The proof is straightforward. 
(i) Let $f$ be a $K$-qc mapping of the half -plane $\mathbb{H}$ on a domain $D$ such that $f(0)=0$, and suppose that $\partial D$ is a $K$-quasi-circle and $\alpha=K^{-1}$. Then $f$ has a $K_{1}$-qc extension to a map $\tilde{f}$ of the complex plane, which by abuse of notation we denote sometimes again by $f$ if there is no possibility of confusion.

\section{Definition 3.3.}

(i) If $z_{1}, z_{2} \in \mathbb{C}^{*}$ by $\theta\left(z_{1}, z_{2}\right)$ we denote the measure of the convex angle between $z_{1}, z_{2}$.

(ii) For $p \in \mathbb{C}$, set

$$
X(z)=X_{p}(z)=\frac{p z}{z-p}, z \in \overline{\mathbb{C}} \quad \text { and } \quad Y=Y_{p}=X^{-1}
$$

(iii) If $f$ is homeomorphism of $\overline{\mathbb{C}}$ onto itself, we define $p=p(f)=f^{-1}(\infty)$.

(iv) If $\gamma$ is an arc in $\mathbb{C}$ and $Z: \gamma \rightarrow \mathbb{C}^{*}$ continuous map by $\Delta_{\gamma} \operatorname{Arg} Z$ we denote the variation of $\operatorname{Arg} Z$ along $\gamma$.

Note that $X$ and $Y$ are Möbius automorphisms of $\overline{\mathbb{C}}$ with the following properties: $Y(z)=-\frac{p z}{z-p}, X(0)=Y(0)=0, X(p)=\infty, X(\infty)=p, Y(p)=\infty$ and $Y(\infty)=$ $-p$. If we set $\breve{f}=f \circ X$, then $f=\breve{f} \circ Y . X_{p}$ and $Y_{p}$ map lines $l_{\beta}=\left\{r e^{i \beta}: r \in \mathbb{R}\right\}$ onto the circles which contain 0 and $p$. Since $Y_{p}$ map the circle $C(0,|p|)$ onto line $L$ which does not contain 0 . If $z_{n}=e^{-i / n} p$ and $z_{n}^{\prime}=e^{i / n} p$, then $\theta\left(z_{n}^{\prime}, z_{n}\right) \rightarrow 0$ and $\theta\left(X z_{n}^{\prime}, X z_{n}\right) \rightarrow \theta_{0}, \theta_{0} \neq 0$, if $n \rightarrow \infty$. This example shows that we need to adapt a version of Proposition 2.8 to hold for the mappings $X_{p}$.

Proposition 3.4. Let $f$ be a $K$-qc mapping of the plane $\overline{\mathbb{C}}$ onto itself, $f(0)=0$, $p=f^{-1}(\infty), \alpha=K^{-1}$ and $r_{0}=|p| / 2$.

(I) (a) Then $f=\breve{f} \circ Y$, where $Y=Y_{p}, \breve{f}$ is $K$-qc mapping of the plane $\overline{\mathbb{C}}$ onto itself, with $\breve{f}(0)=0$ and $\breve{f}(\infty)=\infty$.

(b) If $z_{1}, z_{2} \in \mathbb{C}^{*},\left|z_{1}\right|=\left|z_{2}\right|$ and $\theta \in[0, \pi]$ (respectively $\theta^{*} \in[0, \pi]$ ) is the measure of the convex angle between $z_{1}, z_{2}$ (respectively $\breve{f}\left(z_{1}\right), \breve{f}\left(z_{2}\right)$ ), then $\theta^{*} \leq c \max \left\{\theta^{\alpha}, \theta\right\}$, where $c=c(K)$. In particular, if $\theta \leq 1$, then $\theta^{*} \leq c \theta^{\alpha}$.

(II) If $z_{1}, z_{2} \in \mathbb{C}^{*} \cap B\left(0, r_{0}\right),\left|z_{1}\right|=\left|z_{2}\right|$, then

$$
\theta\left(X z_{1}, X z_{2}\right) \leq\left(1+r_{0}^{-1}\right) \theta\left(z_{1}, z_{2}\right) .
$$

(III) For given $H_{0}^{\prime}=\operatorname{Lyp}(\varepsilon, c, \mu), \varepsilon<r_{0}$, there is $H_{0}=\operatorname{Lyp}\left(\varepsilon_{1}, c_{1}, \mu_{1}\right)$ such that $Y\left(H_{0}\right) \subset H_{0}^{\prime}$.

Proof. (I) Set $\breve{f}=f \circ X$. Since $X(p)=\infty$ and $p=f^{-1}(\infty)$, we have $\breve{f}(\infty)=\infty$. Since $X$ is Möbius automorphism of $\overline{\mathbb{C}}, \breve{f}$ is $K$-qc. By (a) and an application of Proposition 2.8 to $\breve{f}$, (b) follows.

(II) Let $\left|z_{1}\right|=\left|z_{2}\right|=R<r_{0}$. If necessary we can re-numerate points such that $z_{k}=R e^{i t_{k}}, k=1,2, t_{1} \leq t_{2} \leq t_{1}+\pi$ and $l=l\left(z_{1}, z_{2}\right)$ be the circular arc defined by $l(t)=R e^{i t}, t_{1} \leq t \leq t_{2}$. We are going to estimate the variation $\Delta_{l} \operatorname{Arg} T$ and $\Delta_{l} \operatorname{Arg} X$. Since $X(z)=X_{p}(z)=\frac{p z}{z-p}$, we can write 
(i) $\arg X=\arg z-\arg T+\arg p$, where $T=z-p$.

Hence

(ii) $\Delta_{l} \operatorname{Arg} X \leq \Delta_{l} \operatorname{Arg} I d+\Delta_{l} \operatorname{Arg} T$, where $I d$ is the identity map.

Since $T^{\prime} / T=1 / T$, for $z=r e^{i t}$,

$$
(\arg T)_{t}=\operatorname{Im}\left(\frac{T^{\prime}}{T} i r e^{i t}\right) .
$$

For $z \in B\left(0, r_{0}\right)$, we have $\left|(\arg T)_{t}\right| \leq \frac{1}{|z-p|} \leq 1 / r_{0}$, and therefore

$$
\theta\left(T z_{1}, T z_{2}\right) \leq \Delta_{l} \operatorname{Arg} T \leq r_{0}^{-1}\left|t_{2}-t_{1}\right| \text {. }
$$

Hence, by the item (ii), for $\left|z_{1}\right|=\left|z_{2}\right|=R<r_{0}$,

$$
\theta\left(X z_{1}, X z_{2}\right) \leq \Delta_{l} \operatorname{Arg} X \leq\left(1+r_{0}^{-1}\right)\left|t_{2}-t_{1}\right|
$$

(III) We only outline a proof. Set $\zeta=Y(z), \theta=\arg z, \varphi=\arg \zeta, z=x+i y=$ $r e^{i \theta}, \zeta=\xi+i \eta=\rho e^{i \varphi}$. So we define the functions $\theta=\theta(\rho, \varphi), \varphi=\varphi(r, \theta)$, $\rho=\rho(r, \theta)$ and $r=r(\rho, \varphi)$.

Since $Y$ is conformal mapping on $\overline{B\left(0, r_{0}\right)}$ and $Y(0)=0$, by the item (h) in subsection 3.1. we find $\rho(r, \theta) \approx r$ and $r=r(\rho, \varphi) \approx \rho$.

Let $\zeta \in \gamma:=\gamma(\varepsilon, c, \mu), \rho=|\zeta|$ and $z^{\prime}=X(\rho)=r(\rho, 0) e^{i \theta^{\prime}}$, where $\theta^{\prime}=\theta(\rho, 0)$.

Case 1. Suppose that $p=p_{1}+i p_{2}, p_{2}>0$.

Since $X(\infty)=p, X$ maps the coordinate axis $\eta=0$ (in the $\zeta$-plane) onto the circle $K=C\left(i R_{0}, R_{0}\right)$ which contains $p$, where $R_{0}=R_{0}(p)=\frac{|p|^{2}}{2\left|p_{2}\right|}$ depends only on p. $Y_{p}$ maps $B=B\left(i R_{0}, R_{0}\right)$ onto $\mathbb{H}$. Let $K^{\prime}$ be semi circle $y=R_{0}-\sqrt{R_{0}^{2}-x^{2}}$. Then $\theta^{\prime} \approx x(\rho) \preceq r(\rho) \approx r$. By the part (II) of the Proposition, $\theta\left(z, z^{\prime}\right) \preceq \varphi \preceq \rho^{\mu} \preceq r^{\mu}$. Hence, since $\theta \leq \theta\left(z, z^{\prime}\right)+\theta^{\prime}$, we find $\theta \preceq r^{\mu}$ (thus we can choose $\mu_{1}=\mu$ ).

In a similar way we consider:

Case 2. Suppose that $p=p_{0}=p_{1}+i p_{2}, p_{2}<0$. In this case $Y_{p}$ maps $B=$ $B\left(-i R_{0}, R_{0}\right)$ onto $\mathbb{H}^{*}$.

Case 3. $p_{0} \in \mathbb{R}$. In this case $Y_{p}$ maps $\mathbb{H}$ onto itself.

\section{Theorem 3.5.}

(I) Suppose (i): $h$ is a $K$-qc map from $\mathbb{H}$ onto a Lyapunov domain $D$. Then $h$ has a $K_{1}$-qc extension to a map $\tilde{h}$ of the complex plane.

(II) If $h$ satisfies the hypothesis $\mathbb{H}_{q c}^{0}$, then there is a constant $l_{0}=162^{\alpha} k_{1}^{-1}$ which depends on $K_{1}$ and the Kellogg multiplicative constant of $D$ (with respect to $\left.a_{0}=h(i)\right) k_{1}=\underline{k}_{1}\left(D, a_{0}\right)$, such that

(ii): $|h(z)| \leq l_{0}|z|^{1 / K_{1}}$ if $z \in \mathbb{H}$ and $|z| \leq 1$.

(III) If $D$ satisfies the hypothesis (Lyp-0), then there are constants $\varepsilon>0$ and $c>$ 0 such that for $|w|<\varepsilon$ (here $c \varepsilon^{\mu}<\pi$ ) and $w \in \partial D$, either $|\arg (w)|<c|w|^{\mu}$ or $|\pi-\arg (w)|<c|w|^{\mu}$ where $\arg$ is the branch of the argument determined by $-\pi / 2<\arg (w)<3 \pi / 2$ and moreover that set

$D_{0}=D_{0}(\varepsilon)=\operatorname{Lyp}(\varepsilon, c, \mu)=\left\{w: c|w|^{\mu}<\arg (w)<\pi-c|w|^{\mu},|w|<\varepsilon\right\}$ satisfies $D_{0} \subset D$, where $c$ depends only on the Lyapunov multiplicative constant of $\partial D$. 
(iii): We can choose $c=l_{2}=l_{D}^{2}=\frac{\pi}{2} l_{1} b_{\gamma}^{1+\mu}$, where $l_{1}=\operatorname{lyp}(\gamma)$ and $b_{\gamma}=b_{\gamma}^{\text {arc }}$ is the arc-chord constant of $\gamma$.

(IV) Then there is a constant $c_{1}=c_{1}\left(\mu, \varepsilon, c, K_{1}, l_{2},|p|\right)$ such that the region

$$
H_{0}=H_{0}(\varepsilon)=\left\{z: c_{1}|z|^{\mu / K_{1}^{2}}<\arg (z)<\pi-c_{1}|z|^{\mu / K_{1}^{2}},|z|<\left(\varepsilon / l_{0}\right)^{K_{1}}\right.
$$

satisfies

(a) $h\left(H_{0}\right) \subset D_{0}$ and

(b) there are constants $\varepsilon_{2}, c_{2}, \mu_{2}$ such that $D_{0}^{\prime} \subset h\left(H_{0}\right)$, where $D_{0}^{\prime}=\operatorname{Lyp}\left(\varepsilon_{2}, c_{2}, \mu_{2}\right)$.

Note that $H_{0}=H_{0}(\varepsilon)=\operatorname{Lyp}\left(\varepsilon_{1}, c_{1}, \mu_{1}\right)$, where $\mu_{1}=\mu / K_{1}^{2}$ and $\varepsilon_{1}=\left(\varepsilon / l_{0}\right)^{K_{1}}$.

Recall that the hypothesis (i) (together with some technical requirements $h(0)=$ 0 and that $D$ satisfies $(\mathrm{Sp} 0))$ in the theorem is essentially equivalent to the hypothesis $\left(\mathbb{H}_{q c}^{0}\right)$. From the proof below it is clear that the hypothesis (i) implies

(i1): $h$ is a qc mapping of $\mathbb{H}$ onto the quasidisk $D$ (which is much weaker then (i)), and that the statement (I) holds under the hypothesis (i1).

If in addition to (i1), $h(0)=0$ and $0 \in \partial D$, we leave to the interested reader to state and prove a corresponding version of the statement (II). Since $h^{-1}$ is also qc the proof of (IV) of Theorem 3.5 shows that the following holds

$\left(\mathrm{IV}^{\prime}\right)$ : for each special domain of Lyapunov type $X_{0}$ with vertex at 0 , there is a special domain of Lyapunov type $Y_{0}$ with vertex at 0 such that $Y_{0} \subset h\left(X_{0}\right)$. In particular, we can choose $X_{0}=H_{0}$ and $Y_{0}$ to be an elementary Lypunov domain $D_{0}^{-}$such that $D_{0}^{-} \subset h\left(H_{0}\right)$.
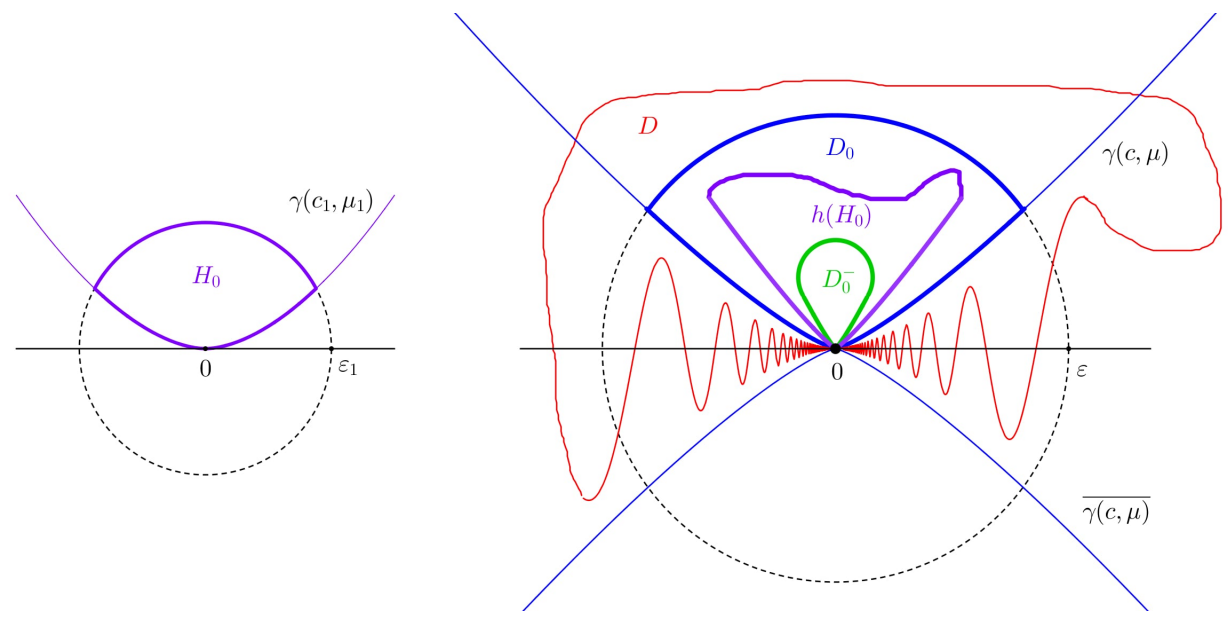

Figure 1.

On the figure 1 the domains $D, D_{0}, D_{0}^{-}, H_{0}$ and $h\left(H_{0}\right)$ are enclosed by lines whose colors are red, blue, green, violet (on the left) and violet (on the right) respectively. 
Proof. Proof of (I). Since $D$ is $C^{1, \mu}, D$ is a quasi-circle and therefore by the item (i) from subsection 3.1. the statement (I) follows.

Proof of (II). We suppose that $a_{0}=h(i)$ is given. Let $B_{1}=B(i ; 1)$ and $R_{1}$ a conformal mapping of $B_{1}$ onto $\mathbb{H}$ such that $R_{1}$ fixes 0 and $i$ and $R_{2}$ a conformal mapping of $D$ onto $B_{1}$ such that $R_{2}(0)=0$ and $R_{2}\left(a_{0}\right)=i$. Set $\underline{h}=R_{2} \circ h \circ R_{1}$. Then $\underline{h}(0)=0, \underline{h}(i)=i, \underline{h}$ maps $B_{1}$ onto itself, $h=R_{2}^{-1} \circ \underline{h} \circ R_{1}^{-1}$. Set $w=$ $h(z), \zeta=R_{1}^{-1}(z), \zeta^{\prime}=R_{2}^{-1}(w)$ and $\zeta^{\prime}=\underline{h}(\zeta)$. Note that $R_{1}^{-1}(\mathbb{H})$ is a disk. By the item (g) in subsection 3.1 , we find $\left|R_{1}^{-1}(z)\right| \leq 2|z|, z \in \mathbb{H}$. Since $D$ is a Lyapunov domain bounded, $\left|R_{2}^{-1}\left(\zeta^{\prime}\right)\right| \leq k_{3}\left|\zeta^{\prime}\right|, \zeta^{\prime} \in B_{1}$, where $k_{3}=\underline{k}_{1}\left(D, a_{0}\right)^{-1}$. It is clear that $\underline{h}$ is $K_{1}$-qc with $\underline{h}(i)=i$. Now an application of Mori's theorem to $\underline{h}$ on $B_{1}$, shows that $\underline{h}$ is $\alpha$-Hölder continuous on $B_{1}$ with a multiplicative constant 16 , where $\alpha=1 / K_{1}$ and in particular $\left|\zeta^{\prime}\right|=|\underline{h}(\zeta)| \leq 16|\zeta|^{\alpha}$. Since $h(0)=0$, there is a constant $l_{0}=l_{0}\left(K_{1}\right)$ such that the part (ii) of the theorem holds: $|h(z)| \leq l_{0}|z|^{1 / K_{1}}$ if $z \in \mathbb{H}$ and $|z| \leq 1$.

Proof of (III). Set $l_{1}=\operatorname{lyp}(\gamma)$. Since $D$ satisfies the hypothesis (Lyp-0), the item (d) in subsection 3.1 holds. By the item (d), there is $\epsilon_{1}>0$ such that the trace of the path $\gamma_{1}$ which is defined in (d) by $v=F(u), u \in\left[-\epsilon_{1}, \epsilon_{1}\right]$, where $F$ is $C^{1}$, is on $\partial D$. Let $\gamma_{2}=\partial D \backslash \operatorname{tr}\left(\gamma_{1}\right)$. Then there is a constant $\epsilon_{2}>0$ such that $\gamma_{2}$ has no points in the disk $B\left(\epsilon_{2}\right)$. Let $L$ be the length of $\partial D$ and $\hat{\gamma}$ a parametrization of the positively oriented boundary $\partial D$ by the arc-length parameter $s$, where $s \in[0, L]$ and $s(0)=0$ (we need arc-length parameter only around 0 ). Set $\hat{\gamma}(s)=u(s)+i v(s)$ and $w=R e^{i \Psi}$. Then

$$
|v(s)|=|v(s)-v(0)|=\left|v^{\prime}\left(s^{1}\right)\right| s \leq c_{1} s^{1+\mu},
$$

where $c_{1}=l_{1}$. Since a quasicircle satisfies the arc-chord condition, we have the following:

(a1) If a $C^{1}$ curve is a quasicircle, then $s \leq c_{2} R$, where $c_{2}=b_{\gamma}=b_{\gamma}^{\text {arc }}$.

Also we can prove the following version of (a1) ${ }^{3}$

(a2) By definition of Lyapunov curve $\hat{\gamma}^{\prime}(t)=1+\epsilon(t)$, where $|\epsilon(t)| \leq c t^{\mu}$. Hence $\hat{\gamma}(s)=\int_{0}^{s} \hat{\gamma}^{\prime}(t) d t$ and therefore there is $\tau=\tau(c, \mu)$ such that $s \leq 2|\hat{\gamma}(s)|$ for $s \leq \tau$.

Hence, there is $\epsilon>0$ such that

(iv) $|\arg \hat{\gamma}(s)|<\frac{\pi}{2}$ for $s \in(0, \epsilon]$ and

(v) $\frac{\pi}{2}<\arg \hat{\gamma}(s)<3 \frac{\pi}{2}$ for $s \in[L-\epsilon, L)$.

We can choose $\epsilon<\epsilon_{2}$. Hence, for $s$ small $(0<s<\epsilon)$, we find

$$
R|\Psi| \leq \frac{2}{\pi} R|\sin (\Psi)|=\frac{2}{\pi} v(s) \leq c_{3} s^{1+\mu}, \quad \text { where } \quad c_{3}=\frac{2}{\pi} c_{1},
$$

and therefore there is a constant $c$ such that

(vi) $|\Psi| \leq c R^{\mu}$, where $c$ is given by the item (iii) in Theorem 3.5 .

Using the mapping $A(w)=-w$ and (vi), we find that

(vi') $|\pi-\Psi| \leq c R^{\mu}$ for $s \in[L-\epsilon, L)$.

From (vi) and (vi'), (III) follows.

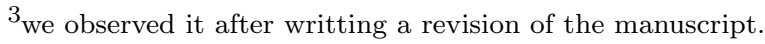


Proof of (IV). We use the notation from Proposition 3.4. Set $\breve{h}=h \circ Y_{p}$, where $p=h^{-1}(\infty) \in \mathbb{C}$. By the statement (I) and easy part of Proposition 3.4 $h=\breve{h} \circ Y_{p}$, and $h$ is $K_{1}$-qc mapping of the plane $\overline{\mathbb{C}}$ onto itself, with $\breve{h}(0)=0$ and $\breve{h}(\infty)=\infty$. We use notation: polar coordinates $\zeta=\rho e^{i \varphi}$ in the $\zeta$-plane and $w=R e^{i \Psi}$ in the $w$-plane. Recall by (III) there is a curve $\gamma=\gamma\left(c, \mu, R_{0}\right)$ in $D$. Set $\gamma_{0}: \Psi=\Psi_{0}(R)=$ $c R^{\mu}, 0 \leq R \leq R_{0}$. Hence there is a part $C_{1}$ of the boundary of $D$ around 0 (say the right half part) which is below $\gamma_{0}$ and which defines the curve $\gamma_{1}$.

Case 1. We first prove for $\breve{h}$. Let $w=R e^{i \Psi} \in \gamma_{0}$ and let $w^{\prime}=R e^{i \Psi^{\prime}}$ be the intersection of the circle $T_{R}$ with $\gamma_{1}$. Then $\theta\left(w, w^{\prime}\right) \leq\left(|\Psi|+\left|\Psi^{\prime}\right|\right) \leq 2|\Psi|$. Set $\zeta=\breve{h}^{-1}(w)$ and $\zeta^{\prime}=\breve{h}^{-1}\left(w^{\prime}\right)$. Since $\gamma_{0}$ is the right half of $\gamma=\gamma(c, \mu), \zeta^{\prime}>0$ and $\varphi=\theta\left(z, z^{\prime}\right)$. Hence using the quasihyperbolic metric $k$ on $\mathbb{C}^{*}$ (Proposition 2.8), we have

$\varphi \leq \kappa_{2} \Psi^{\alpha}$, where $\alpha=1 / K_{1}$. Since, by (II), $R \leq c \rho^{\alpha}$, we find $\varphi \leq \kappa_{2}\left(\Psi_{0}\left(c \rho^{\alpha}\right)\right)^{\alpha}$ and therefore we get $\varphi \leq \kappa_{3} r^{\alpha^{2} \mu}$. Thus we find:

(vii) the curve $\breve{h}^{-1}\left(\gamma_{0}\right)$ is below the curve $\gamma\left(\kappa_{3}, \alpha^{2} \mu\right)$.

Note that if a curve satisfies $\varphi \leq c \rho^{\mu}$, then it is is below the curve $\gamma(c, \mu)$. Recall that we set $\mu_{1}=\mu / K_{1}^{2}$ and $\varepsilon_{1}=\left(\varepsilon / l_{0}\right)^{K_{1}}$. Note that $\gamma_{0}$ is the right half of $\gamma=\gamma(c, \mu)$ and that in a similar way as above we conclude that

(viii) $\breve{h}^{-1}(\gamma(c, \mu))$ is below the curve $\gamma\left(c_{1}, \mu_{1}\right), \rho<\varepsilon_{1}$.

By the part (ii) of the theorem, $h\left(B\left(\varepsilon_{1}\right)\right) \subset B(\varepsilon)$ and it is readable that it yields (a). Since $\breve{h}^{-1}$ is also qc (a) implies (b). Thus we have proved (IV) for $\breve{h}$ with $c_{1}=\kappa_{3}$.

Case 2. Proof for $h$. By Case 1 , there is $H_{0}^{\prime}$ such that $\breve{h}\left(H_{0}^{\prime}\right) \subset D_{0}$. By Proposition 3.4 there is $H_{0}$ such that $Y\left(H_{0}\right) \subset H_{0}^{\prime}$ and it completes proof. Thus we have proved (a).

Let us prove that (a) implies (b). Namely, since $h^{-1}$ is also qc, by (a) there is $D_{0}^{\prime}$ such that $h^{-1}\left(D_{0}^{\prime}\right) \subset H_{0}$ and therefore $D_{0}^{\prime} \subset h\left(H_{0}\right)$.

3.2. Global approximation. Concerning the previous theorem, note that $\mu_{1} \leq \mu$ and $\varepsilon_{1} \leq \varepsilon$, and in particular, one can derive (see (IV')):

(a) there is $\underline{\epsilon}=\underline{\epsilon}(\varepsilon, c)<\varepsilon_{1}$ such that $h\left(L^{1}\right) \subset L^{\prime} \subset D_{0}$, where $L^{\prime}=\operatorname{Lyp}^{-}(\varepsilon, c)$ is $\mu$-Lyapunov and $L^{1}=\operatorname{Lyp}^{-}(\underline{\epsilon}, c)$ is $\mu_{1}$-Lyapunov and $L^{1} \subset L^{\prime}$. Hence, since $h^{-1}$ is also qc, $h^{-1}\left(L^{1}\right) \subset L^{\prime}$ and therefore $L^{1} \subset h\left(L^{\prime}\right)$.

(b) In a similar way, there is $\underline{\epsilon}^{1} \leq \underline{\epsilon}$ and $\mu_{2} \leq \mu_{1}$ such that $h^{-1}\left(L_{-}^{1}\right) \subset L^{1}$, where $L_{-}^{1}=\operatorname{Lyp}^{-}\left(\underline{\epsilon}^{1}, c\right)$ is $\mu_{2}$-Lyapunov.

Hence we derive:

(IVa) If $h$ satisfies the hypothesis $\mathbb{H}_{q c}^{0}$, then $L_{-}^{1} \subset h\left(L^{1}\right) \subset D_{0}$.

Note that it is easy to transfer Theorem 3.5 to the setting of the unit disk. Now we show that the corresponding version of it holds with $\mathbb{U}$ instead of $\mathbb{H}$.

We first need a version of $\left(I^{\prime}\right)$ for $\mathbb{U}$ with special Lyapunov convex domains. Note that $H_{0}$ has two cusps. In this subsection by $D_{0}$ we denote the set defined in Theorem 3.5 .

Definition 3.6 $\left(\operatorname{lyp}(D)_{b}\right)$. Here we define $\underline{A}_{0}, R_{a}, T_{b}$ and $h_{a}$. 
QUASICONFORMAL AND HQC MAPPINGS BETWEEN LYAPUNOV JORDAN DOMAINS 15

(i) Consider the conformal mapping $A_{0}=\underline{A}_{0}$ defined by $\underline{A}_{0}(z)=\frac{4 i-z}{4 i+z} ; \underline{A}_{0}$ maps $\mathbb{H}$ onto $\mathbb{U}$ such that $\underline{A}_{0}(0)=1$ and $\underline{A}_{0}(-4 i)=\infty$.

(ii) For $a=e^{i \alpha} \in \mathbb{T}$ define $R_{a}(z)=e^{i \alpha} z$, and for $b \in \partial D$ if the unit inner normal $n_{b}=e^{i \beta}$ at $b$ exists, we define $T_{b}(w)=\underline{T}_{b}(w)=-i e^{i \beta} w+b$, and

(iii) if $D$ satisfies the hypothesis (Lyp-0), we define $\hat{D}_{b}=T_{b}\left(\hat{D}_{0}\right)$, where $\hat{D}_{0}$ is defined in the item (A) below. If we wish to indicate that $\hat{D}_{b}$ is an elementary Lyapunov domain we use notation $\operatorname{lyp}(D)_{b}$.

(iv) For $a \in \mathbb{T}$, set $h_{a}=h_{a}^{b}:=\underline{T}_{b}^{-1} \circ h \circ R_{a}, a \in \mathbb{T}$, where $b=h(a)$, and let $\hat{h}=h \circ A_{0}$ and $\hat{h}_{a}=h_{a} \circ A_{0}$.

(v) Next suppose that $D$ satisfies the hypothesis (Lyp-0) (see Definition 1.5).

We will prove that(see also Proposition 3.8):

(A) there is $\varepsilon_{2}>0$ such that $\hat{D}_{0} \subset T_{b}^{-1}(D)$ for every $b \in \partial D$, where $\hat{D}_{0}=$ $\operatorname{Lyp}^{-}\left(\epsilon_{2}, c\right)$.

(vi) In addition to (v) suppose that $h$ is a qc mapping of $\mathbb{U}$ onto $D$ and $h(1)=0$ (that is $h$ satisfies the hypothesis $\mathbb{U}_{q c}^{0}$ ).

Then $\hat{h}$ is a qc mapping of $\mathbb{H}$ onto $D$ with $\hat{h}(0)=0$.

It seems useful to consider the following properties, which is an immediate corollary of (IVa):

(B) If $h$ satisfies the hypothesis $\mathbb{U}_{q c}^{0}$, there are corresponding elementary Lyapunov domains $H^{1} \subset \mathbb{H}$ and $D_{-}^{1} \subset D$ with vertex at 0 such that $D_{-}^{1} \subset$ $\hat{h}\left(H^{1}\right) \subset \hat{D}_{0}$. See Proposition 3.8 for a stronger result.

(vii) Further set $U_{1}=A_{0}\left(H^{1}\right)$ and $U_{-}^{1}=A_{0}\left(D_{-}^{1}\right)$ and set $U_{a}=R_{a}\left(U_{1}\right)$.

Note that $H^{1}$ is a special $\mu_{1}$-Lyapunov. Thus we have

(C) $U_{-}^{1} \subset h\left(U_{1}\right) \subset \hat{D}_{0}$.

In order to state a corresponding form of (IVa) for $\mathbb{U}$, it is convenient to call $V=$ $A_{0}(L)$ an elementary domain if $L$ is elementary (see also Proposition 3.4. Now, by (IVa), it is clear that we have:

(IVb) If $h$ satisfies the hypothesis $\mathbb{U}_{q c}^{0}$ and $L$ is an elementary Lyapunov domain with vertex at 0 in $\mathbb{H}$, then there are elementary Lyapunov domains $V_{1}$ and $V_{-}^{1}$ in $\mathbb{U}$ with vertex at 1 such that $V_{-}^{1} \subset h\left(V_{1}\right) \subset L$.

(D) Now, we also suppose that $h$ satisfies the hypothesis $\mathbb{U}_{q c}^{0}$. Recall by the item (i) from subsection 3.1, then $h$ has a $K_{1}$-qc extension to a map $\tilde{h}$ of the complex plane.

We can choose $p=\tilde{p}$ such that $h(\tilde{p})=\infty$ and $|\tilde{p}| \geq 3$. Set $\hat{p}=A_{0}^{-1}(\tilde{p}), \tilde{p}_{\alpha}=$ $h_{a}^{-1}(\infty)$ and $\hat{p}_{\alpha}=A_{0}^{-1}\left(\tilde{p}_{\alpha}\right)$. Check that $\tilde{p} \notin h(B(0,2))$ and therefore $|\hat{p}| \geq 2$.Hence, since $\tilde{p}_{\alpha}=h_{a}^{-1}(\infty)=e^{-i \alpha} \tilde{p}$, we find

$(D 0): h_{a}^{-1}(\infty) \notin h(B(0,2))$ and therefore, $\left|\hat{p}_{\alpha}\right| \geq 2$ for every $a=e^{i \alpha} \in \mathbb{T}$.

Note that $T_{b} \circ h_{a}=h \circ R_{a}$ and that $h$ satisfies the hypothesis $\mathbb{U}_{q c}^{0},(U-1)$ if and only if $\hat{h}$ satisfies $\mathbb{H}_{q c}^{0},(H-0)$ respectively.

In addition, we need a property of $C^{1}$ domains. Suppose that domain $D$ is $u v$-plane. 


\section{Lemma 3.7.}

(i) Suppose that a $C^{1}$ domain $D$ satisfies the hypothesis ( $\left.\mathrm{Sp} 0\right)$.

(I) Then there is $r>0$ such that for each $w \in \partial D, \underline{T}_{w}^{-1}(\partial D) \cap B(0, r)$ is a graph with respect to $u v$-coordinates.

Proof. Let $L$ be the length of $\partial D$ and $\hat{\gamma}:[0, L] \rightarrow \partial D$ a parametrization of the positively oriented boundary $\partial D$ by the arc-length parameter $s$. We also write $w=\hat{\gamma}(s)$, where $s \in[0, L]$ and $s(0)=0$. Here there is the function $s=s(w)$ which is the inverse of the function $w=\hat{\gamma}(s)$ and which maps $\partial D$ onto $[0, L]$. Since $\hat{\gamma}^{\prime}$ is continuous on $[0, L]$, it is uniformly continuous on $[0, L]$. The function $s=s(w)$ is continuous on $\partial D$ and hence $C(w)=\hat{\gamma}^{\prime}(s(w))$ is continuous on $\partial D$ and uniformly continuous on $\partial D$. Therefore there is $r_{2}>0$ such that

(ii) $\left|\arg \hat{\gamma}^{\prime}\left(s_{2}\right)-\arg \hat{\gamma}^{\prime}\left(s_{1}\right)\right|<\pi / 8$ for $\left|w_{2}-w_{1}\right| \leq r_{2}$, where $w_{k}=\hat{\gamma}\left(s_{k}\right)$, $k=1,2$.

Let us to prove (I) for $r=r_{2} / 2$. Contrary, suppose that (I) is not true. Then for some $w_{0} \in \partial D, D\left(w_{0}, r\right):=(\partial D) \cap B\left(w_{0}, r\right)$ is not a graph with respect to coordinates determined by unit vectors $\hat{\gamma}^{\prime}(w)$ and $n_{w}$. Hence there are two points $w_{1}$ and $w_{2}$ in this set such that $w_{1} w_{2}$ is parallel to the normal $n_{w_{0}}$ of $\partial D$ at $w_{0}$. Therefore there is $w_{3}$ in this set such that $\gamma^{\prime}(s)$ at $w_{3}$ is parallel to the normal $n_{w_{0}}$. This contradicts (ii). Thus we have (I).

Using the approach in the proof of statement (III), (IV), (IV') of Theorem 3.5. (IVb) and (iii), we can prove

\section{Proposition 3.8.}

(a): Suppose that $D$ satisfies the hypothesis (Lyp-0).

(I): there is an elementary Lyapunov domains $\hat{D}_{0}$ in $D$ with vertex at 0 such that $\hat{D}_{0} \subset \underline{T}_{b}^{-1}(D)$.

(b): In addition to (a) suppose that $h: \mathbb{U} \rightarrow D$ is a qc homeomorphism.

(IIa): Then there is a Lyapunov domain $\hat{U}_{1}$ in $\mathbb{U}$ with vertex at 1 such that for every $a \in \mathbb{T}, h_{a}\left(\hat{U}_{1}\right) \subset \hat{D}_{0}$.

(IIb): In addition, there is an elementary Lyapunov domain $D_{0}^{-}=\operatorname{Lyp}\left(\varepsilon_{0}^{-}, c_{0}, \mu\right)$ in $D$ with vertex at 0 such that $D_{0}^{-} \subset h_{a}\left(\hat{U}_{1}\right)$ for every $a \in \mathbb{T}$.

(III): $D_{0}^{-} \subset h_{a}\left(\hat{U}_{1}\right) \subset \hat{D}_{0}$

Note that in general $h_{a}(\mathbb{U})$ is not a fixed domain for $a \in \mathbb{T}$ and therefore we need first to consider the part (I) and then the part (II).

Proof. (I). $D_{b}$ satisfies the hypothesis (Lyp-0) for every $b \in \partial D$. Consider the family $\underline{\mathcal{D}}:=\left\{D_{b}=\underline{T}_{b}^{-1}(D): b \in \partial D\right\}$. For $b \in \partial D$ define $\varepsilon(b)$ to be maximum of $\varepsilon$ for which $\operatorname{Lyp}\left(\varepsilon, l_{2}(D)\right) \subset \overline{D_{b}}$, where $l_{2}(D)$ is the second Lyp-constant. By Lemma 3.7 . there is $r>0$ such that $D_{b} \cap B(r)$ is a graph with respect to $u v$-coordinates for each $b \in \partial D$. Since all domains $D_{b}, b \in \partial D$, have the same Lyapunov multiplicative constants, using (iii) and the approach in the proof (III) of Theorem 3.5, we can prove that there is $\varepsilon_{0}>0$ such that $\varepsilon(b) \geq \varepsilon_{0}$ for all $b \in \partial D$, and therefore an elementary Lyapunov domain $\hat{D}_{0}$ such that $\hat{D}_{0} \subset D_{b}$ for every $b \in \partial D$ and (I) 
follows.

In addition, it seems that we can prove that the function $\varepsilon(b)=\varepsilon_{D}(b)$ is continuous with respect to $b$.

(IIa): Recall that we use the notation $a_{0}=h(0)$ and $\hat{h}_{a}=h_{a} \circ \underline{A}_{0}$. Let $\omega_{b}$ be a conformal mapping of $D_{b}$ onto $\mathbb{U}$ such that $\omega_{b}\left(\underline{T}_{b}^{-1}\left(a_{0}\right)\right)=0$ and $\omega^{b}=\omega_{b}$ 。 $\underline{T}_{b}^{-1}$. Since $\omega^{b}(0)=0$, by the Kellogg-Warshawski theorem there are two positive constants $l_{1}$ and $l_{2}$ such that $l_{1} \leq\left|\omega^{\prime}(w)\right| \leq l_{2}, w \in D$. Since $\underline{T}_{b}^{-1}$ is a euclidean isometry, we have $\left|\left(\underline{T}_{b}^{-1}\right)^{\prime}\right|=1$ on $D$, and therefore $l_{1} \leq\left|\omega_{b}^{\prime}(w)\right| \leq l_{2}, w \in D_{b}$. Hence, since $\omega_{b} \circ h_{a}$ maps $\mathbb{U}$ onto itself, $\omega_{b} \circ h_{a}(0)=0$ and $h_{a}(1)=0$ there is a constant $l^{0}$ which depends on $K$ and the Kellogg multiplicative constant of $D$ (with respect to $a_{0}$ ), such that for all $a \in \mathbb{T},\left|h_{a}(z)\right| \leq l^{0}|z-1|^{1 / K}$ if $|z| \leq 1$.

Using $\underline{A}_{0}$ we can get the corresponding result for $\hat{h}_{a}$ : there is a constant $l_{0}$ which depends on $K_{1}$ and the Kellogg multiplicative constant of $D$, such that for all $a \in \mathbb{T}$,

(iv): $\left|\hat{h}_{a}(z)\right| \leq l_{0}|z|^{1 / K_{1}}$ if $|z| \leq 1, \operatorname{Im} z \geq 0$.

The functions $\hat{h}_{a}, a \in \mathbb{T}$, are $K_{1}$-qc. By (iv), an application of (IVb) to the functions $\hat{h}_{a}, a \in \mathbb{T}$, and the statement $(D 0)$ (from subsection 3.2), and the item (iii) of Proposition 3.4 with $r_{0}=1$ to the functions $\hat{h}_{a}, a \in \mathbb{T}$, show that there is a Lyapunov domain $\hat{H}_{0}$ in $\mathbb{H}$ with vertex at 0 such that $\hat{h}_{a}\left(\hat{H}_{0}\right) \subset \hat{D}_{0}$. Set $\hat{U}_{1}=A_{0}\left(\hat{H}_{0}\right)$. It yields the proof of (II).

(IIb): Using (IVb), since the corresponding parameters are the same for $h$ and $h_{a}$, one can get (III).

(III) It is clear that (IIa) and (IIb) can be stated as (III).

It is convenient to introduce the following notation:

(v): For $b \in \partial D$, set $D_{b}^{-}=\operatorname{lyp}(D)_{b}^{-}=\underline{T}_{b}\left(D_{0}^{-}\right)$and for $a \in \mathbb{T}, \hat{U}_{a}=R_{a}\left(\hat{U}_{1}\right)$. If we wish to indicate that $D_{b}^{-}$is an elementary Lyapunov domain we use notation $\operatorname{lyp}(D)_{b}^{-}$.

Now using euclidean isometry $\underline{T}_{b}$ it is easy to get the corresponding results of the property (III) of Proposition 3.8 for domains with vertexes at $b$. Namely, by the property (III) of Proposition 3.8 we have $\underline{T}_{b}\left(D_{0}^{-}\right) \subset \underline{T}_{b}\left(h_{a}\left(\hat{U}_{1}\right)\right) \subset \underline{T}_{b}\left(\hat{D}_{0}\right)$. By the definitions $\underline{T}_{b} \circ h_{a}\left(\hat{U}_{1}\right)=h \circ R_{a}\left(\hat{U}_{1}\right)=h\left(\hat{U}_{a}\right)$ and therefore the part (I) of the next theorem follows. By (L1) (see the introduction) we get the part (II). So we have the crucial result:

Theorem 3.9. Suppose that $D$ is a Lyapunov domain and $h: \mathbb{U} \rightarrow D$ is a $q c$ homeomorphism. Then

(I) For every $a \in \mathbb{T}, \operatorname{lyp}(D)_{b}^{-} \subset h\left(\hat{U}_{a}\right) \subset \operatorname{lyp}(D)_{b}$, where $b=h(a)$.

(II) If $w-b$ is in the direction of the normal vector $n_{b}$ then, $d_{b}(w) \approx|w-b|$ if $\varepsilon_{2}=\varepsilon_{2}\left(c_{2}, \mu_{2}\right)$ is a small enough constant.

\section{Proof that $h$ IS CO-LIPSChitz}

Here we give proof of co-Lip property: 
Theorem 4.1. Suppose $h: \mathbb{U} \rightarrow D$ is a hqc homeomorphism, where $D$ is a Lyapunov domain with $C^{1, \mu}$ boundary, i.e. belonging to $\mathcal{D}_{1}$. Then $h$ is co-Lipschitz.

We first need a few results mentioned in the introduction.

Theorem 4.2 (Theorem 1.3, 24]).

(i) Suppose that $h$ is a euclidean harmonic complex valued mapping from the unit ball $\mathbb{B} \subset \mathbb{R}^{n}$ onto a bounded domain $D=h(\mathbb{B})$, which contains the ball $B\left(h(0) ; R_{0}\right)$ and there is a half space $H_{b}$ which touches the point $b \in \partial D$ such that $D=h(\mathbb{B}) \subset H_{b}$. Then

(I) $d(h(z), b) \geq(1-|z|) \bar{c}_{n} R_{0}, \quad z \in \mathbb{B}$, where $\bar{c}_{n}=\frac{1}{2^{n-1}}$.

Sometimes, we refer to this result as a version of Harnack's lemma.

In 24 we stated this result under the condition that the domain $D=h(\mathbb{B})$ is convex. But, a slight modification of the proof of Theorem 1.1 [24] (planar case) shows that the theorem holds under the hypothesis (a).

Proof of $(I)$. We only outline an argument. To $b \in \partial D$ we associate a nonnegative harmonic function $u=u_{b}$. Let $\Lambda_{b}$ be the boundary of $H_{b}$ and let $n=n_{b} \in T_{b} \mathbb{R}^{n}$ be a unit vector such that $\Lambda_{b}$ is defined by $\left(w-b, n_{b}\right)=0$. By hypothesis, $\Lambda_{b}$ is a supporting hyper-plane such that $\left(w-b, n_{b}\right) \geq 0$ for every $w \in \bar{D}$. Define $u(z)=\left(h(z)-b, n_{b}\right)$ and $d_{b}=d\left(h(0), \Lambda_{a}\right)$. Then $u(0)=\left(h(0)-b, n_{b}\right)=d\left(h(0), \Lambda_{a}\right)$. Let $b_{0} \in \Lambda_{a}$ be the point such that $d_{b}=\left|h(0)-b_{0}\right|$. Then from the geometry it is clear that $d_{a} \geq R_{0}$, etc (one can follow the proof from [24]).

Proposition 4.3. Suppose that $h$ is a euclidean harmonic mapping from the Lyapunov domain $G$ into a domain $\Omega$ and

(i) there is a half space $H_{b}$ which touches a point $b \in \partial \Omega$ such that $h(G) \subset H_{b}$.

Then $d(h(z), b) \succeq d_{G}(z), z \in G$.

Note if $h: \mathbb{U} \rightarrow D$ satisfies hypothesis $U$-1, in general a point $b \in \partial D$ does not satisfy the hypothesis (i). We use elementary Lyapunov domain described in Proposition 3.8 to apply this proposition.

Proof. Let $\phi: \mathbb{U} \rightarrow G$ be a conformal mapping and $h_{1}=h \circ \phi$. Application of Koebe's theorem to $\phi$ and Theorem 4.2 on $h_{1}: \mathbb{U} \rightarrow G$ yield the result.

Now we illustrate relation between the circles and special Lyapunov curves and then prove Lemma 4.4 .

If $M(0, d), d>0$, then the circle $C$ with center at $M$ and radius $d$ is given by the equation $x^{2}+(y-d)^{2}=d^{2}$ and the half -circle $C^{-}$with $y=d-\left(d^{2}-x^{2}\right)^{1 / 2}$. Hence $d-y=\left(d^{2}-x^{2}\right)^{1 / 2}=d\left(1-x^{2} / d^{2}\right)^{1 / 2}=d\left(1-x^{2} / 2 d^{2}+o\left(x^{2}\right)=x^{2} / 2 d+o\left(x^{2}\right)\right.$ and therefore $y=\frac{1}{2 d} x^{2}+o\left(x^{2}\right)$. The graph of the curve $\gamma(c, \mu ; \epsilon)$, where $c=1 / d, \epsilon=d$ is above the half -circle $C$.

Lemma 4.4. For $c>0,0<\mu<1$, and $x_{0}>0$, let the curve $C$ be defined by (the curve $C$ is defined in Example 2.7 and denoted by $f(c, \mu)$ )

(1): $y=C(x)=c x^{1+\mu},|x|<x_{0}$, and

(2): let $M(0, d), d>0$, be a point and $d^{\prime}$ the distance from $M$ to the graph of the curve (1). 
(I) Then $d^{\prime} \succeq d$ and

(a) there is an $\epsilon^{0}>0$ such that if $d \leq C\left(\epsilon^{0}\right)$, then $d \leq 2 d^{\prime}$.

(b) For $\epsilon^{0}$ we can choose the positive solution of the equation $c^{2}(1+\mu) x_{1}^{2 \mu}=1$.

Proof. Let $d^{\prime}=\left|M-M^{\prime}\right|$, where $M^{\prime}\left(x_{1}, y_{1}\right)$. Since $y^{\prime}(x)=c(1+\mu) x^{\mu}$, we find that $k=\frac{d-y_{1}}{x_{1}}=\left(c(1+\mu) x_{1}^{\mu}\right)^{-1}$. Hence, $d-y_{1}=\frac{1}{c(1+\mu)} x_{1}^{1-\mu}$ and $d=\frac{1}{c(1+\mu)} x_{1}^{1-\mu}+y_{1}=$ $\frac{1}{c(1+\mu)} x_{1}^{1-\mu}\left(1+c^{2}(1+\mu) x_{1}^{2 \mu}\right)$. Hence, $d \leq 2 d^{\prime}$ if $\left(1+c^{2}(1+\mu) x_{1}^{2 \mu}\right) \leq 2$ (that is if $\varepsilon_{2}=\varepsilon_{2}\left(c_{2}, \mu_{2}\right)$ is a small enough constant).

We are now ready to finish the proof. We will apply Proposition 3.8 and notation used there, and Theorem 4.2 Further chose a fixed positive real number $x_{0} \in \hat{U}_{1}$.

(E) Set $H(a)=h_{a}\left(x_{0}\right)=T_{b}^{-1}\left(h\left(x_{0} a\right)\right), w^{\prime}=T_{b}^{-1}(w), d_{0}\left(w^{\prime}\right)=\operatorname{dist}\left(w^{\prime}, \partial D_{0}^{-}\right)$ and $d^{0}(a)=d_{0}\left(T_{b}^{-1}\left(h\left(x_{0} a\right)\right)\right)$, where $b=h(a)$. It is straightforward to check that $H$ and $d^{0}$ are continuous function with respect to $a \in \mathbb{T}$. Hence there is $s_{0}>0$ such that $B\left(H(a), s_{0}\right) \subset D_{0}^{-}$and therefore we conclude

(E0) If $h$ satisfies the hypothesis $\mathbb{U}_{q c}^{0}$, there is a constant $s_{0}>0$ which does not depend of $a$ such that $B\left(h\left(x_{0} a\right), s_{0}\right) \subset D_{b}^{-}, a \in \mathbb{T}$.

Recall that for $a=e^{i \alpha} \in \mathbb{T}$, we let $\phi=\phi_{a}$ be the conformal mapping of $\mathbb{U}$ onto $U_{a}$ such that $\phi_{a}(0)=x_{0} e^{i \alpha}$ and $F=F(h)=F_{a}=h \circ \phi_{a}$. Let $b \in \partial D, w-b=\epsilon n_{b}$, $\epsilon \leq \epsilon_{0}$. Then $w \in D_{b}^{-}$.

$$
\begin{aligned}
\text { Set } a & =h^{-1}(b), z=h^{-1}(w), z^{\prime}=\phi_{a}^{-1}(z), d\left(z^{\prime}\right)=1-\left|z^{\prime}\right|, r^{\prime}=\left|z^{\prime}\right| \\
d_{a}(z) & =\operatorname{dist}\left(z, \partial \hat{U}_{a}\right), d_{b}(w)=\operatorname{dist}\left(w, \partial D_{b}^{-}\right) \quad \text { and } \quad d_{0}\left(w^{\prime}\right)=\operatorname{dist}\left(w^{\prime}, \partial D_{0}^{-}\right) .
\end{aligned}
$$

Let $H_{b}$ be the half plane which contains $\hat{D}_{b}$ and touches $D$ at $b$. Since $F_{a}$ maps $\mathbb{U}$ into $H_{b},\left(F_{a}\left(z^{\prime}\right)-b, n_{b}\right)$ is non-negative in $z^{\prime} \in \mathbb{U}$, and by a version of Harnack's estimate, $\left|F_{a}\left(z^{\prime}\right)-b\right| \geq s^{0}\left(1-r^{\prime}\right)$, i.e. $|w-b| \geq s^{0} d\left(z^{\prime}\right)$. Hence (1) $|w-b| \succeq d\left(z^{\prime}\right)$.

From Lemma 4.4 (the geometric property of the domain $D_{b}^{-}$), we find $|w-b| \preceq$ $d_{b}(w)$ and therefore

(3) $\quad d_{b}(w) \succeq d\left(z^{\prime}\right)$.

Set $\epsilon_{0}:=\min \left\{\varepsilon_{0}^{-}, \epsilon^{0}\right\}$ and $D\left(\epsilon_{0}\right):=\left\{w \in D: d(w, \partial) \geq \epsilon_{0}\right\}$. Then there is $r_{0}, r_{1} \in(0,1)$ such that $D\left(\epsilon_{0}\right) \subset h\left(B\left(r_{0}\right)\right)$ and $r^{\prime}=\left|z^{\prime}\right| \geq r_{1}$ implies $|z| \geq r_{0}$.

By Theorem 4.2 (note here that the estimate in this theorem depends on $R_{0}$ and it is independent of $h$ ) and (E0), we conclude

(E1) there is $s_{1}>0$ which is independent of $z^{\prime}$ such that $d_{b}(w) \geq s_{1} d\left(z^{\prime}\right)$, $r^{\prime}=\left|z^{\prime}\right| \geq r_{1}$.

Recall that $d_{b}^{\prime}(w)=\operatorname{dist}\left(w, Y_{a}\right)$, where $Y_{a}:=h\left(\hat{U}_{a}\right)$. We now estimate $\Lambda_{h}(z)$. Using the fact that $h\left(\hat{U}_{a}\right)=Y_{b} \supset D_{b}^{-}$, we find first that $d_{b}^{\prime}(w) \geq d_{b}(w)$ and therefore

$$
\Lambda_{h}(z) \succeq \frac{d_{b}^{\prime}(w)}{d_{a}(z)} \geq \frac{d_{b}(w)}{d_{a}(z)} .
$$

Since $\phi_{a}(\mathbb{U})=\hat{U}_{a}$ and $\hat{U}_{a}$ is a Lyapunov domain of a fixed shape, $d_{a}(z) \approx d\left(z^{\prime}\right)$.

Combining this with (3), we conclude $\lambda_{h}(z) \approx \Lambda_{h}(z) \geq s_{2}>0$, where $s_{2}>0$ is a constant. Hence, using (E1), we conclude 
(F) $\lambda_{h}(z) \approx \Lambda_{h}(z) \geq s_{2}>0,|z| \geq r_{0}$, where $s_{2}>0$ is a constant independent of $z$.

It is clear that there is a constant $s_{3}>0$ such that

(F1) $\lambda_{h}(z) \approx \Lambda_{h}(z) \geq s_{3}>0, z \in B\left(r_{0}\right)$.

By $(\mathrm{F})$ and $(\mathrm{F} 1)$, there is a constant $s_{4}>0$ such that $\lambda_{h}(z) \approx \Lambda_{h}(z) \geq s_{4}>0$, $z \in \mathbb{U}$.

Hence it is readable that $h$ is co-Lip on $\mathbb{U}$.

Remark 4.5. By application of Proposition 4.3 onto the restriction of $h$ on $\hat{U}_{a}$ one can also get a proof of Theorem 4.1.

\section{Further COMments AND RELATED RESUlts}

We briefly discus the connection with the Radó-Kneser-Choquet theorem (shortly RKC-Theorem) and hyperbolic-harmonic mappings; it will be the subject of further investigations.

Quasiconformal euclidean-harmonic mappings are bi-Lipschitz with respect to the quasi-hyperbolic metric, cf. [24, 19] (Proposition 2.3 here). It turns out that, as in the euclidean case, quasiconformal hyperbolic-harmonic mappings are bi-Lipschitz with respect to the hyperbolic metric, cf. Wan 31] and of Markovic [20.

Very recently, concerning the initial Schoen Conjecture (and more generally the Schoen-Li-Wang conjecture) Markovic made a major breakthrough. In 21, Markovic used the result of $\mathrm{Li}$ and Tam that every diffeomorphism of $\mathbb{S}^{2}$ admits a harmonic quasiisometric extension to show that every quasisymmetric homeomorphism of the circle $\partial \mathbb{H}^{2}$ admits a harmonic quasiconformal extension to the hyperbolic plane $\mathbb{H}^{2}$. This proves the initial Schoen Conjecture.

In particular, concerning complex valued harmonic functions, Kalaj and the second author, shortly KM-approach, study lower bounds of the Jacobian, cf. [25, 26] and references cited there. The corresponding results for harmonic maps between surfaces were previously obtained by Jost and Jost-Karcher 10, 11. We refer to this result as the JK- result (approach). G. Alessandrini and V. Nesi prove necessary and sufficient criteria of invertibility for planar harmonic mappings which generalize a classical result of H. Kneser, also known as the Radó-Kneser-Choquet theorem (RKC-Theorem), cf. [1]. Note only here that in the planar case the JKresult is reduced to Theorem RKC. Kalaj [13] also has extended the Rado-ChoquetKneser theorem to mappings between the unit circle and Lyapunov closed curves with Lipschitz boundary data and essentially positive Jacobian at the boundary (but without restriction on the convexity of the image domain). The proof is based on the extension of the Rado-Choquet-Kneser theorem by Alessandrini and Nesi [2] and an approximation scheme is used in it. Motivated by an approach described in Kalaj's Studia paper [13] and using the continuity of so called $E$-function, the second author found a new proof of Kalaj's result, cf. 25, 26.

Acklowedgment. We are indebted to M. Svetlik for helping us in preparation this manuscript. In particular we thank him for making the Figure 1. We are indebted to the referee, N. Mutavdžić and D. Kalaj for useful comments which improved the exposition. 


\section{REFERENCES}

[1] G. Alessandrini, V. Nesi, Invertible Harmonic mappinngs, beyond Kneser, arXiv:0712.3840v2 [math.AP] 8 Apr 2008

[2] G. Alessandrini, V. Nesi: Invertible harmonic mappings, beyond Kneser, Ann. Scuola Norm. Sup. Pisa, Cl. Sci. (5) VIII (2009), 451-468.

Errata Corrige, Ann. Scuola Norm. Sup. Pisa, Cl. Sci.(2) XVII (2017) 815-818.

[3] G. Alessandrini, V. Nesi, Estimates for the dilatation of $\sigma$-harmonic mappings, arXiv:1412.4248 1 [math.AP],13 Dec 2014, Rendiconti di Matematica, Serie VII, Volume 35, Roma (2014), 215 - 226

[4] L. V. Ahlfors, Lectures on Quasiconformal Mappings: Second Edition, with additional chapters by C. J. Earle and I. Kra, M. Shishikura, J. H. Hubbard, University Lecture Series Volume: 38; 2006; 162 pp - See more at: http://bookstore.ams.org/ulect-38\#sthash.zmUmCeS7.dpuf

[5] C. J. Bishop, Bilipschitz approximations of quasiconformal maps, Ann. Acad. Sci. Fenn. Math.27(1): 97-108 (2002).

[6] F.W. Gehring and B.G. Osgood, Uniform domains and the quasi-hyperbolic metric, J. Anal. Math. 36(1979), 50-74.

[7] J. T. Gill, S. Rohde, A note on quasiconformal maps with Hölder continuous dilatation, CRM Preprint Series number 1147

[8] G. M. Goluzin: Geometric function theory, Nauka Moskva 1966 (Russian).

[9] E. Heinz, On one-to-one harmonic mappings, Pac. J. Math. 9 (1959), 101-105.

[10] J. Jost, Harmonic Maps Between surfaces, Springer-Verlag Berlin Heidel berg New York Tokyo, 1984.

[11] J. Jost, Two-dimensional Geometric Variational Problems, John Wiley \& Sons, 1991.

[12] D. Kalaj, Quasiconformal harmonic mapping between Jordan domains, Math. Z. Volume 260, Number 2, 237-252, 2008.

[13] D. KALAJ, Invertible harmonic mappings beyond Kneser theorem and quasiconformal harmonic mappings, Studia mathematica (ISSN:0039-3223), Volume 207, Issue 2, Dec. 2011, Page(s) $117-136$

[14] D. Kalaj, Harmonic mappings and distance function, Ann. Scuola Norm. Sup. Pisa Cl. Sci.

(5) Vol. X (2011), 669-681.(arXiv:1011.3012)

[15] D. Kalaj, M. Mateljević, (K,K')-quasiconformal Harmonic Mappings, Potential Anal. (2012) 36:117-135 DOI 10.1007/s11118-011-9222-4

[16] D. Kalaj, M. Mateljević, Inner estimate and quasiconformal harmonic maps between smooth domains, Journal d'Analise Math. 100. 117-132, (2006).

[17] D. Kalaj, M. Mateljević, Quasiconformal Harmonic Mappings and Generalizations, Proceedings of the ICM2010 Satellite Conference International Workshop on Harmonic and Quasiconformal Mappings (HQM2010), Editors: D. Minda, S. Ponnusamy, and N. Shanmugalingam J. Analysis Volume 18 (2010), 239-260

[18] M. Knežević, M. Mateljević, On the quasi-isometries of harmonic quasiconformal mappings, J. Math. Anal. Appl. 334(1), 404-413 (2007)

[19] V. Manojlović, Bi-Lipschicity of quasiconformal harmonic mappings in the plane, Filomat 23, No. 1, 2009, 85-89.

[20] V. Markovic, Harmonic diffeomorphisms of noncompact surfaces and Teichmller spaces, J. Lond. Math. Soc. (2) 65(1), 103-114 (2002)

[21] V. Markovic, Harmonic maps and the Schoen Conjecture, March 7, 2015, http://www.its.caltech.edu/ markovic/M-schoen.pdf

[22] O. Martio, On harmonic quasiconformal mappings, Ann. Acad. Sci. Fenn., Ser. A I 425 (1968), $3-10$.

[23] M. Mateljević, Estimates for the modulus of the derivatives of harmonic univalent mappings, Proceedings of International Conference on Complex Analysis and Related Topics (IX ${ }^{t h}$ Romanian-Finnish Seminar, 2001), Rev Roum Math Pures Appliq (Romanian Journal of Pure and Applied mathematics) 47 (2002) 5-6, 709 -711. 
[24] M. Mateljević, Distortion of harmonic functions and harmonic quasiconformal quasiisometry, Revue Roum. Math. Pures Appl. Vol. 51 (2006) 5-6, 711-722.

[25] M. Mateljević, The Lower Bound for the Modulus of the Derivatives and Jacobian of Harmonic Injective Mappings, Filomat 29:2(2015), 221-244, Doi: 10.2298/FIL1502221M

[26] M. Mateljević, Invertible harmonic and Harmonic quasiconformal mappings, Filomat 29:9 (2015) 1953-1967

[27] D. Partyka, K. Sakan: On bi-Lipschitz type inequalities for quasiconformal harmonic mappings. Ann. Acad. Sci. Fenn., Math. 32, 579-594 (2007)

[28] D. Partyka, K. Sakan: Quasiconformal and Lipschitz Harmonic Mappings of the Unit Disk Onto Bounded Convex Domains. In submission.

[29] M. Pavlović, Boundary correspondence under harmonic quasiconformal homeomorphisms of the unit disc, Ann. Acad. Sci. Fenn., Vol 27, (2002), 365-372.

[30] M. Vuorinen, Conformal geometry and quasiregular mappings, Lecture Notes in Mathematics, 1988th Edition

[31] T. Wan, Constant mean curvature surface, harmonic maps, and universal Teichmller space, J. Differ. Geom. 35(3), 643-657 (1992)

[32] S.E. Warschawski, On the higher derivatives at the boundary in conformal mapping, Trans. Am. Math. Soc, 38(2), 310340 (1935) 